\title{
Effects of Grounding (Earthing) on Massage Therapists: An Exploratory Study
}

\author{
Gaétan Chevalier ${ }^{1}$, Sheila Patel ${ }^{1,2}$, Lizabeth Weiss ${ }^{2}$, Christopher Pruitt ${ }^{1}$, \\ Brook Henry33, Deepak Chopra1,2, Paul J. Mills ${ }^{1}$ \\ ${ }^{1}$ Department of Family Medicine and Public Health, University of California, San Diego, CA, USA \\ ${ }^{2}$ The Chopra Center for Wellbeing, Carlsbad, CA, USA \\ ${ }^{3}$ Department of Psychiatry, University of California, San Diego, CA, USA \\ Email: dlbogc@sbcglobal.net
}

How to cite this paper: Chevalier, G., Patel, S., Weiss, L., Pruitt, C., Henry, B., Chopra, D. and Mills, P.J. (2018) Effects of Grounding (Earthing) on Massage Therapists: An Exploratory Study. Health, 10, 228-250. https://doi.org/10.4236/health.2018.102019

Received: January 21, 2018

Accepted: February 25, 2018

Published: February 28, 2018

Copyright () 2018 by authors and Scientific Research Publishing Inc. This work is licensed under the Creative Commons Attribution International License (CC BY 4.0).

http://creativecommons.org/licenses/by/4.0/

cc) (i) Open Access

\begin{abstract}
It is well known that massage therapists often develop a number of health problems relatively early on in their career. A preliminary study showed that grounding massage therapists during their work may alleviate some of the health problems they encounter. A doubled-blind randomized controlled trial was designed to examine the effects of working and sleeping grounded for 4 weeks on massage therapists' blood viscosity, stress (through HRV), inflammation (IFN- $\gamma$, IL-6, TNF- $\alpha$, and hsCRP) and oxidative stress (MPO and MDA) biomarkers. The results show stress reduction as measured by heart rate, respiratory rate and hear rate variability (HRV) and a lowering effect on blood viscosity that lasted for at least one week after ungrounding, with systolic blood viscosity becoming significantly lower at the end of the study. Inflammation markers (IFN- $\gamma$, TNF- $\alpha$, and hsCRP) increased rapidly, within one week, after ungrounding. The findings suggest that grounding is beneficial for massage therapists in multiple domains relevant to health and wellbeing.
\end{abstract}

\section{Keywords}

Earthing, Grounding, Heart Rate Variability (HRV), Blood Viscosity, Inflammatory Biomarkers, Oxidative Stress

\section{Introduction}

\subsection{Earthing (Grounding)}

Earthing, or grounding, are terms interchangeably used to describe the condition of being in direct contact with the earth (ground). Examples of grounding 
include walking bare feet on grass or moist soil, swimming in the ocean or a lake, or using specially designed equipment such as grounding sheets and mats that conductively connects individuals to the ground when they are indoors. These grounding products are typically connected to the earth using a building's electrical grounding system or by planting a metal rod directly into the soil.

Several benefits of being grounded have been reported. They include improvement in sleep, reduction of chronic and acute inflammation, decrease in physiological as well as psychological stress, normalization of cortisol level, decrease muscle damage during exercise, decrease blood viscosity, improvement in blood circulation, increase wellness feeling and positive mood, and normalization of muscles tension [1] [2] [3].

\subsection{Massage Therapists' Burnout and Pain}

It is well known that massage therapists often develop a number of health problems relatively early on in their career [4]. Most of these health problems stem from repetitive motions and overuse injuries and include tendonitis, painful fingers, hands, wrists, elbows and shoulders, carpal tunnel syndrome, and a host of other work-related injuries [5]. A commonality behind these injuries is chronic inflammation, which is associated with chronic pain [6] [7] [8]. These realities of daily work life, and the lack of effective relief, prompt many massage therapists to leave their profession prematurely; they are "burnt out". Burnout is a frequently used term to describe the cumulative wear and tear on the body and psyche that lead dedicated massage therapists to leave their profession [9] [10].

A previously completed pilot study examined whether grounding massage therapists during their massage work could help alleviate the stress and pain they experience [5]. Based on the promising positive findings obtained in that pilot study, this larger exploratory study sought to extend those findings by including a larger set of assessments and grounding during the therapists' night sleep as well as while they performed massages.

\section{Methods}

\subsection{Bioethics Committee}

This study protocol was approved and supervised by BioMed IRB, a San Diego based independent IRB (http://biomedirb.com/). The study was performed at the Chopra Center for Wellbeing in Carlsbad, California.

\subsection{Subjects}

Sixteen (16) healthy massage therapists employed at The Chopra Center for Wellbeing in Carlsbad, California with at least one year of experience and with no diagnosis of a major disease participated. These participants were trained by the Chopra Center on all massages methods used by the center; they all received the same training. 


\subsection{Study Design and Procedures}

Participants were explained the research protocol and signed the consent form approved by the IRB. A study staff member then asked each massage therapist to complete the Chopra Center medical form. The staff member also explained how to fill the Heart Monitor Log Form (to be filled at the end of every day of work). All massage techniques used at the Chopra Center were included in this project. The staff member also gave to each participant a Grounded Beauty Tummy Band (earthing.com, Thousand Palms, CA) with two number coded grounding cords and with instructions on how to use the Grounded Beauty Tummy Band at home and how to connect the second cord to the grounded mat in the massage room. Instructions were given on where and when to go to give a blood sample. Blood samples were taken by a licensed phlebotomist at the beginning of the study, at the end of week 5 and at the end of week 6 .

At the end of the first week, a study staff member met with massage therapists to collect the log forms, make sure they were filled properly and that the protocol was followed. The staff member also took back the two number coded grounding cables and replaced them with two cables coded with different numbers. The process of exchanging cables for another set with a different number code was repeated every week and it was established to help maintain the double-blind nature of the study. Heart Monitor Log forms were collected at the end of every week at the same time a new set of cables was given except at the end of week 6 when no cables were given (that was the end of their participation in the study). Finally, a study staff member recorded any comment from the massage therapists related to their participation in the study.

A double-blind Randomized Controlled Trial (RCT) procedure was used based on the stepped wedge design. In a stepped wedge design, an intervention is rolled-out sequentially to the trial participants (either as individuals or clusters of individuals) over a number of time periods. The order in which the different individuals or clusters receive the intervention is determined at random and, by the end of the random allocation, all individuals or groups will have received the intervention. Stepped wedge designs incorporate data collection at each point where a new group (step) receives the intervention [11] [12]. In this project there were two interventions: grounding and sham-grounding. Each intervention was identified by a number coded band around the cord, the signification of the number code (grounded or sham grounded) not being known to the massage therapists, study staff and researchers. The number code was only known to the person who prepared the cables for this study. Except for the number coding band, all cords looked alike but the modified cords did not allow electrical conduction from the earth to the mat. The number coding information was kept secret until after the last week of the last cluster was completed and after all the log forms and blood sample results were received by the principal investigator (PI).

Therapists were randomly assigned to a cluster, or cohort. The duration of each cohort's participation was 6 weeks and was divided as follows: 
- First week of participation subjects were not grounded.

- The next 4-week period they were grounded.

- The last week they were not grounded.

\subsection{Grounding Equipment and Method}

Grounding (earthing) was accomplished in two ways: first using a grounding mat placed on the floor around the massage table in the massage room and through the use of a grounding tummy band that participants used at home during sleep. A study staff checked that the ground outlets used by massages therapists at the Chopra Center were working properly and massage therapists were given a ground checker to verify that their home grounding system was working properly. They were blinded to when they were or were not grounded, receiving a different "grounding" cord at the start of each week of the study. On weeks 1 and 6, therapists were given number coded cords that did not ground them (placebo cord) while they were given proper number coded grounding cords (active grounding cords) at weeks 2, 3, 4 and 5. In order for them not to suspect which week they were grounded or not, new number coded cords were given every week.

\subsection{Blood Viscosity}

Blood viscosity is an important factor affecting the ability of blood to circulate in the blood vessels. It is a factor contributing to cardiovascular disease [13], a risk factor for type 2 diabetes mellitus [14], and a predictor of decline in general cognition [15]. Since blood is a non-Newtonian fluid, its viscosity varies greatly during a cardiac cycle. Blood viscosity also varies with the anatomical configuration of an artery. For example, blood viscosity at the aorta is different from that at the coronary artery because the sheer rate is different at the two locations. Blood viscosity is high at low shear rates and low at high shear rate. Normal blood viscosity varies from $3.8 \mathrm{cP}(38 \mathrm{mP})$ at high shear rate $\left(300 \mathrm{~s}^{-1}\right)$ to about 20 $\mathrm{cP}(200 \mathrm{mP})$ at low shear rate $\left(1 \mathrm{~s}^{-1}\right)$. Blood viscosity at high shear rate is called systolic blood viscosity (SBV), analogous to systolic blood pressure, while blood viscosity at low shear rate is termed diastolic blood viscosity (DBV) [16].

Historically, accurately measuring blood viscosity was a difficult task that required the use of rotational viscometers that allowed testing for blood viscosity at a single shear rate. It was a time-consuming process and technically demanding [17] [18]. For this study, a state-of-the-art instrument resolved all the problems of previous methods. Invented by Dr. Young Cho, a fluid dynamics expert and professor of mechanical engineering at Drexel University, this instrument (the Hemathix Blood Analyzer SCV-200 by Health Onvector, Camden, NJ), measures blood viscosity over a wide range of shear rates representative of the cardiac cycle in a single continuous measurement. For this study, EDTA lavender tubes filled with 3 milliliters of blood were shipped to Onvector for blood viscosity analysis. These blood samples were drawn at the beginning of week 1 , and 
at the end of week 5 and 6 by a certified phlebotomist and immediately placed on ice. For each blood sample, SBV and DBV were measured and analyzed (see Results section).

\subsection{Biomarkers}

Blood samples, which were collected in EDTA, were drawn at the beginning of week 1 , and at the end of weeks 5 and 6 by a certified phlebotomist and immediately placed on ice. Samples were transported from the study site to the UC San Diego Clinical Research Biomarker Laboratory by study personnel, at which time the samples were processed and immediately stored at $-80{ }^{\circ} \mathrm{C}$ until time of biological assay. IL6, TNF- $\alpha$, IFN- $\gamma$ were determined by use of a commercially available ELISA (Meso Scale Discovery, Rockville, Maryland). MPO (myeloperoxidase) and hsCRP (high-sensitivity C-Reactive Protein) were determined by use of a commercially available ELISA (Research \& Diagnostic Systems, Inc., Minneapolis, Minnesota). MDA (malondialdehyde) was determined by use of a commercially available ELISA (MyBioSource, San Diego, California). Blood specimens were analyzed in blinded pairs, and the lab technician running the assays was blinded to the identity of each sample. All subject's samples were run within the same assay plates. Intra-assay and inter-assay coefficients of variance were less than $7 \%$.

\subsection{Heart Rate Variability}

Hearth Rate Variability (HRV) is an established measure of autonomic nervous system modulation of the cardiovascular system [19]. In this study, heart rate was recorded from a portable HRV monitor (Zephyr Biopatch sensor, Medtronic, Annapolis, MD). Subjects were given the Biopatch before their first day of work and they were instructed to wear the Biopatch sensor on the first work day of week 1, and on the last work day of week 5 and week 6 . They were instructed to start the Biopath (i.e. start the recording of their HR data) at 8 am and to keep it recording until at least 30 minutes after they finish working on their last client. HRV was calculated for four 10-minute periods for each of these 3 days. The 4 periods were: the first 10 minutes after they put on the Biopatch, the last $10 \mathrm{mi}$ nutes before they work on their first patient, the first 10 minutes after they worked on the last patient and the last 10 minutes of the day. Participants were allowed to engage in free movement during the intervals at the beginning and end of the work day (sitting, standing, or walking). They were standing during the intervals immediately before and after the massages.

Variables calculated for each of the 10-minute periods from raw data included:

- Average Heart Rate (HR);

- Average Respiratory Rate (RR);

- SDNN: the standard deviation of the interval between normal heart beats (the $\mathrm{NN}$ interval) 
- LF: Low frequency component $(0.04-0.15 \mathrm{~Hz})$ of the Power Spectral Density

- HF: High frequency component $(0.15 \mathrm{~Hz}-0.4 \mathrm{~Hz})$ of the Power Spectral Density

- LF/HF: Ratio of low to high frequency bands of the Power Spectral Density. These variables were measured and analyzed according to the standards established by the Task Force of the European Society of Cardiology and the North American Society of Pacing and Electrophysiology [20] using the VivoSense software platform (VivoSense, Inc., Newport Beach, CA). Digitized ECG data were analyzed to detect the R-wave peaks of the QRS complex. The power spectrum density (PSD) of the HRV signal was assessed using the nonparametric Welch periodogram method with Fast Fourier Transform (FFT) [21]. Since participants were allowed to move during intervals of HR/HRV recording (e.g., not required to remain stationary and sitting in a chair) we utilized a multi-step process to identify and remove artifacts from the signal that may be generated by motor activity. First, the beat-to-beat ECG waveform was visually inspected and missing or unidentified R-peaks were manually relabeled. RR interval artifacts were subsequently removed with linear spline interpolation. Third, an automated VivoSense artifact marking algorithm was also applied to identify and remove ectopic beats and spurious HR (excluding HR above 220 or below 30 bpm) before HRV data output. We developed an Heart Monitor Log Form to have a written record of exactly at what time in the morning participants put the BioPatch on and at what time they took it off.

\subsection{Statistical Analysis}

Statistical calculations were performed using NCSS/PASS 2000 edition licensed with Dawson's book: Basic \& Clinical Biostatistics, Third Edition, McGraw-Hill, New York, 2001. Parametric mean comparisons were performed using t-test for differences between means (paired and equal variance) or Aspin-Welch Unequal-Variance test (unequal variance). When the parametric assumption did not hold, statistical tests used included: Wilcoxon Signed-Rank Test for difference in means (paired), Quantile (Sign) Test (paired), and Wilcoxon Rank-Sum Test for difference in means (non-paired). Chi-square was used to determine significance between cohorts' gender distribution. We considered the usual 0.05 as the threshold for statistical significance.

\section{Results}

\subsection{Age and Gender Distribution}

Table 1 presents age, gender and body mass index (BMI) characteristics of the therapists who participated. Female massage therapists represented $69 \%$ of the participants. Average age between genders was comparable: the age range for females was between 30 and 55 years and for males between 34 and 54 years. While cohort selection was randomized, Cohort A had an even number of male and female participants while Cohort B had only one male participant. 
Chi-square analysis, however, indicated there was not a significant gender difference between the two cohorts $(\chi$ statistic $=2.62, \mathrm{p}=0.11)$. Age was not significantly different between the two Cohorts $(\mathrm{t}=0.89, \mathrm{p}=0.38)$. However, BMI was significantly higher for males compared to females $(t=3.79, \mathrm{p}=0.002)$.

\subsection{Blood Viscosity}

Blood viscosity results are presented in Table 2 for cohorts and Table 3 for

Table 1. Age, gender and BMI characteristics of the participants.

\begin{tabular}{|c|c|c|c|c|c|c|c|c|}
\hline$A+B$ & $\mathrm{~N}$ & $\%$ & A & $\mathrm{N}$ & $\%$ & B & $\mathrm{N}$ & $\%$ \\
\hline Gender & & & Gender & & & Gender & & \\
\hline Female & 11 & $68.8 \%$ & Female & 4 & $25.0 \%$ & Female & 7 & $43.8 \%$ \\
\hline Male & 5 & $31.3 \%$ & Male & 4 & $25.0 \%$ & Male & 1 & $6.3 \%$ \\
\hline Total & 16 & $100.0 \%$ & Total & 8 & $50.0 \%$ & Total & 8 & $50.0 \%$ \\
\hline Age & Years & BMI & Age & Years & BMI & Age & Years & BMI \\
\hline Female & & & Female & & & Female & & \\
\hline Ave (SD) & $42.5(8.0)$ & $20.7(1.5)$ & Ave (SD): & $39.8(2.2)$ & $20.3(1.7)$ & Ave (SD): & $44.1(9.7)$ & $20.8(1.4)$ \\
\hline Male & & & Male & & & Male & & \\
\hline Ave (SD) & $43.4(7.5)$ & $24.8(3.0)$ & Ave (SD): & $42.5(8.3)$ & $24.8(3.5)$ & Ave (SD): & 47 (N/A) & $25.1(\mathrm{~N} / \mathrm{A})$ \\
\hline Combined & & & Combined & & & Combined & & \\
\hline Ave (SD) & $42.8(7.6)$ & 22.0 & Ave (SD): & $41.1(5.8)$ & $22.6(3.5)$ & Ave (SD): & $44.5(9.1)$ & $21.4(2.0)$ \\
\hline
\end{tabular}

Table 2. Blood viscosity cohort results.

\begin{tabular}{|c|c|c|c|c|c|c|}
\hline \multirow{2}{*}{ Cohort } & \multicolumn{2}{|c|}{ WEEK 1} & \multicolumn{2}{|c|}{ WEEK 5} & \multicolumn{2}{|c|}{ WEEK 6} \\
\hline & Systolic & Diastolic & Systolic & Diastolic & Systolic & Diastolic \\
\hline \multicolumn{7}{|l|}{ A } \\
\hline Ave (SD) & $3.77(0.20)$ & $9.91(0.94)$ & $3.68(0.25)$ & $9.68(0.95)$ & $3.63(0.34)$ & $9.43(1.50)$ \\
\hline Week 1 & & & 0.106 & 0.191 & 0.038 & 0.145 \\
\hline Week 5 & & & & & 0.273 & 0.271 \\
\hline \multicolumn{7}{|l|}{ B } \\
\hline Ave (SD) & $3.48(0.28)$ & $8.20(1.61)$ & $3.61(0.28)$ & $8.89(1.49)$ & $3.43(0.29)$ & $8.00(1.90)$ \\
\hline Week 1 & & & 0.051 & 0.053 & 0.344 & 0.243 \\
\hline Week 5 & & & & & 0.035 & 0.082 \\
\hline \multicolumn{7}{|l|}{$A+B$} \\
\hline Ave (SD) & $3.62(0.28)$ & $9.06(1.55)$ & $3.65(0.26)$ & $9.28(1.28)$ & $3.54(0.32)$ & 8.76 (1.79) \\
\hline Week 1 & & & 0.353 & 0.195 & 0.134 & 0.192 \\
\hline Week 5 & & & & & 0.055 & 0.111 \\
\hline \multicolumn{7}{|l|}{ A vs B } \\
\hline $\mathrm{p}$ value & 0.018 & 0.012 & 0.215 & 0.104 & 0.091 & 0.047 \\
\hline
\end{tabular}


Table 3. Blood viscosity gender results.

\begin{tabular}{ccccccc}
\hline A + B & \multicolumn{2}{c}{ WEEK 1 } & \multicolumn{2}{c}{ WEEK 5 } & \multicolumn{2}{c}{ WEEK 6 } \\
\hline Females & Systolic & Diastolic & Systolic & Diastolic & Systolic & Diastolic \\
\hline Ave (SD): & $3.50(0.22)$ & $8.47(1.40)$ & $3.57(0.20)$ & $8.85(1.16)$ & $3.42(0.23)$ & $8.13(1.51)$ \\
W1 & & & 0.175 & 0.136 & 0.143 & 0.172 \\
W5 & & & & & 0.004 & 0.031 \\
\hline Males & Systolic & Diastolic & Systolic & Diastolic & Systolic & Diastolic \\
\hline Ave (SD): & $3.90(0.17)$ & $10.34(1.06)$ & $3.82(0.31)$ & $10.23(1.04)$ & $3.86(0.34)$ & $10.50(1.37)$ \\
W1 & & & 0.246 & 0.405 & 0.386 & 0.398 \\
W5 & & & & & 0.122 & 0.158 \\
F vs M & & & & & & \\
p value: & 0.003 & 0.009 & 0.066 & 0.041 & 0.011 & 0.017 \\
\hline
\end{tabular}

genders in centipoise $(\mathrm{cP})$. Numbers in red represent statistically significant differences between blood viscosity results. Numbers in blue represent non-significant results that may be of interest for future research (see Discussion section for more details). Table 2 shows that for Cohort A there was a significant decrease in SBV at week 6 compared to week $1(\mathrm{p}=0.038)$. For Cohort $\mathrm{B}$, there was a significant decrease in SBV at week 6 compared to week $5(\mathrm{p}=0.035)$. When comparing Cohort A and Cohort B for the same week, it can be seen from Table 2 that Cohort A started week 1 with a significantly higher average blood viscosity than Cohort B for both SBV and DBV ( $p=0.018$ and $p=0.012$, respectively). Also, DBV was significantly lower for Cohort B at week $6(p=0.047)$.

Table 3 presents results between genders combining both cohorts. It can be observed from this table that for females both SBV and DBV decreased significantly at week 6 compared to week $5(\mathrm{p}=0.004$ and 0.031$)$. There was no significant result for males. Female vs. male mean comparisons show that males had significantly higher SBV and SDV than females for all weeks (except for SBV at week 5).

\subsection{Blood Biomarkers}

Table 4 presents biomarker results for the concentration levels of IFN- $\gamma$, IL-6, TNF- $\alpha$, hsCRP (markers of inflammation) and MPO and MDA (markers of oxidative stress). For Cohort A, IFN- $\gamma$ average concentration was significantly higher at week 6 compared to week 1 and week 5 ( $p=0.035$ and 0.040 , respectively). For Cohort B, TNF- $\alpha$ average concentration was higher at week 6 compared to week 1 ( $p=0.023$ ), while hsCRP average concentration was significantly higher at week 6 compared to week 1 and week 5 ( $\mathrm{p}=0.034$ and 0.019 , respectively). Looking at both cohorts combined, it can be seen that TNF- $\alpha$ average concentration was higher at week 6 compared to week $1(p=0.047)$ while hsCRP average concentration was significantly higher at week 6 compared to week 5 and week 1 ( $\mathrm{p}=0.015$ and 0.017 , respectively). 
Table 4. Average concentration of blood biomarkers for each cohort with statistical results.

\begin{tabular}{|c|c|c|c|c|c|c|c|c|}
\hline Cohort & Week & Visit Date & IFN- $\gamma(\mathrm{pg} / \mathrm{mL})$ & IL-6(pg/mL) & TNF-alpha(pg/mL) & $\mathrm{hsCRP}(\mathrm{mg} / \mathrm{L})$ & $\mathrm{MPO}(\mathrm{ng} / \mathrm{mL})$ & $\operatorname{MDA}(\mathrm{ng} / \mathrm{mL})$ \\
\hline \multicolumn{9}{|l|}{ A } \\
\hline Ave (SD) & 1 & $2016 / 11 / 14$ & $5.91(3.61)$ & $0.30(0.21)$ & $1.36(0.57)$ & $0.81(0.69)$ & $20.11(7.34)$ & $9.54(14.68)$ \\
\hline Ave (SD) & 5 & $2016 / 12 / 14$ & $7.45(3.12)$ & $0.68(0.65)$ & $1.31(0.42)$ & $1.49(2.74)$ & $21.40(7.22)$ & $4.80(1.44)$ \\
\hline Ave (SD) & 6 & $2016 / 12 / 22$ & $10.39(5.88)$ & $0.48(0.54)$ & $1.42(0.59)$ & $2.26(2.92)$ & $21.68(6.20)$ & $8.40(9.28)$ \\
\hline W1 vs W5 & & & 0.156 & 0.118 & 0.389 & 0.227 & 0.156 & 0.074 \\
\hline W1 vs W6 & & & 0.035 & 0.107 & 0.273 & 0.096 & 0.191 & 0.191 \\
\hline W5 vs W6 & & & 0.040 & 0.219 & 0.230 & 0.109 & 0.363 & 0.180 \\
\hline \multicolumn{9}{|l|}{ B } \\
\hline Ave (SD) & 1 & $2017 / 1 / 9$ & $5.75(2.11)$ & $0.42(0.28)$ & $0.89(0.15)$ & $0.75(0.68)$ & $15.44(6.70)$ & $7.32(4.14)$ \\
\hline Ave (SD) & 5 & $2017 / 2 / 9$ & $7.10(5.36)$ & $0.55(0.50)$ & $1.11(0.27)$ & $0.61(0.46)$ & $16.46(6.11)$ & $9.67(9.01)$ \\
\hline Ave (SD) & 6 & $2017 / 2 / 16$ & $4.55(1.65)$ & $0.62(0.54)$ & $0.98(0.13)$ & $1.35(0.70)$ & $14.26(4.42)$ & $10.14(8.92)$ \\
\hline W1 vs W5 & & & 0.167 & 0.260 & 0.098 & 0.353 & 0.331 & 0.241 \\
\hline W1 vs W6 & & & 0.078 & 0.191 & 0.023 & 0.034 & 0.306 & 0.421 \\
\hline W5 vs W6 & & & 0.068 & 0.188 & 0.124 & 0.019 & 0.218 & 0.297 \\
\hline \multicolumn{9}{|l|}{$A+B$} \\
\hline Ave (SD) & 1 & & $5.83(2.86)$ & $0.35(0.24)$ & $1.17(0.48)$ & $0.78(0.66)$ & $17.78(7.21)$ & $8.50(10.79)$ \\
\hline Ave (SD) & 5 & & $7.29(4.15)$ & $0.61(0.55)$ & $1.21(0.36)$ & $1.05(1.94)$ & $18.93(6.95)$ & $7.40(6.27)$ \\
\hline Ave (SD) & 6 & & $7.66(5.25)$ & $0.55(0.52)$ & $1.22(0.48)$ & $1.81(2.09)$ & $18.22(6.50)$ & $9.21(8.83)$ \\
\hline W1 vs W5 & & & 0.094 & 0.207 & 0.227 & 0.090 & 0.229 & 0.210 \\
\hline W1 vs W6 & & & 0.180 & 0.232 & 0.047 & 0.015 & 0.138 & 0.316 \\
\hline W5 vs W6 & & & 0.395 & 0.339 & 0.385 & 0.017 & 0.363 & 0.341 \\
\hline
\end{tabular}

Table 5. Statistical results of blood biomarker concentrations between cohorts for each week.

\begin{tabular}{ccccccc}
\hline A vs B & IFN- $\gamma(\mathrm{pg} / \mathrm{mL})$ & IL-6 $(\mathrm{pg} / \mathrm{mL})$ & TNF-alpha $(\mathrm{pg} / \mathrm{mL})$ & hsCRP $(\mathrm{mg} / \mathrm{L})$ & MPO $(\mathrm{ng} / \mathrm{mL})$ & $\mathrm{MDA}(\mathrm{ng} / \mathrm{mL})$ \\
\hline Week 1 & 0.914 & 0.281 & 0.038 & 0.874 & 0.205 & 0.707 \\
Week 5 & 0.877 & 0.684 & 0.275 & 0.414 & 0.162 & 0.138 \\
Week 6 & 0.027 & 0.728 & 0.094 & 0.435 & 0.021 & 0.719 \\
All Ws & 0.054 & 0.451 & 0.009 & 0.681 & 0.003 & 0.291 \\
\hline
\end{tabular}

Table 5 presents results for the same biomarkers but comparing Cohort A with Cohort B for different weeks. The table shows that IFN- $\gamma$ average concentration was significantly higher for Cohort A at week $6(\mathrm{p}=0.027$; refer to Table 4 for average concentration values). The same table also shows that TNF- $\alpha$ average concentration was significantly higher for Cohort A at week $1(\mathrm{p}=0.038)$, and also when comparing all weeks combined $(\mathrm{p}=0.009)$. Similarly, MPO average concentration was significantly higher for Cohort $A$ at week $6(p=0.021)$, and also when comparing all weeks combined $(\mathrm{p}=0.003)$.

Table 6 presents results for the same biomarkers for combined cohorts by 
gender. For females, IFN- $\gamma$ average concentration was significantly higher at week 5 compared to week 1 ( $\mathrm{p}=0.032$ ), TNF- $\alpha$ average concentration was significantly lower at week 1 compared to week 5 and week $6(\mathrm{p}=0.048$ and 0.016 , respectively), hsCRP average concentration was significantly higher at week 6 compared to week 1 and week 5 ( $\mathrm{p}=0.007$ and 0.004 , respectively), and MPO average concentration was higher at week 6 compared to week $1(\mathrm{p}=0.047)$. There are no significant results for male participants.

Table 7 presents results for the same biomarkers however, this time, comparing Cohort A female participants with Cohort B female participants for different

Table 6. Genders statistical results of blood biomarker concentrations for each week.

\begin{tabular}{|c|c|c|c|c|c|c|c|}
\hline$F(A+B)$ & Week & IFN- $\gamma(\mathrm{pg} / \mathrm{mL})$ & IL-6 (pg/mL) & TNF-alpha (pg/mL) & hsCRP (mg/L) & MPO (ng/mL) & $\operatorname{MDA}(\mathrm{ng} / \mathrm{mL})$ \\
\hline Ave (SD) & 1 & $5.50(2.52)$ & $0.33(0.15)$ & $0.94(0.15)$ & $0.69(0.59)$ & $16.08(5.87)$ & $6.01(3.80)$ \\
\hline Ave (SD) & 6 & $5.76(2.98)$ & $0.47(0.47)$ & $1.04(0.21)$ & $2.04(2.28)$ & $17.88(7.00)$ & $8.32(7.39)$ \\
\hline W1 vs W5 & & 0.032 & 0.107 & 0.048 & 0.248 & 0.117 & 0.377 \\
\hline W1 vs W6 & & 0.366 & 0.297 & 0.016 & 0.007 & 0.047 & 0.196 \\
\hline W5 vs W6 & & 0.145 & 0.091 & 0.274 & 0.004 & 0.483 & 0.148 \\
\hline$M(A+B)$ & Week & IFN- $\gamma(\mathrm{pg} / \mathrm{mL})$ & IL-6(pg/mL) & TNF-alpha(pg/mL) & hsCRP(mg/L) & $\mathrm{MPO}(\mathrm{ng} / \mathrm{mL})$ & $\mathrm{MDA}(\mathrm{ng} / \mathrm{mL})$ \\
\hline Ave (SD) & 1 & $6.56(3.72)$ & $0.38(0.20)$ & $1.68(0.58)$ & $0.98(0.83)$ & $21.51(9.13)$ & $13.48(18.12)$ \\
\hline Ave (SD) & 6 & $12.89(7.02)$ & $0.75(0.67)$ & $1.69(0.72)$ & $0.94(1.02)$ & $19.14(5.69)$ & $11.66(13.08)$ \\
\hline W1 vs W5 & & 0.772 & 0.552 & 0.360 & 0.375 & 0.731 & 0.189 \\
\hline W1 vs W6 & & 0.173 & 0.272 & 0.875 & 0.953 & 0.625 & 0.450 \\
\hline W5 vs W6 & & 0.152 & 0.250 & 0.364 & 0.458 & 0.719 & 0.443 \\
\hline
\end{tabular}

Table 7. Females statistical results of blood biomarker concentrations between cohorts for each week.

\begin{tabular}{|c|c|c|c|c|c|c|c|c|}
\hline A vs $B(F)$ & Week & Visit Date & $\mathrm{IFN}-\gamma(\mathrm{pg} / \mathrm{mL})$ & IL-6 (pg/mL) & TNF-alpha (pg/mL) & hsCRP (mg/L) & MPO (ng/mL) & MDA (ng/mL) \\
\hline \multicolumn{9}{|l|}{ A } \\
\hline Ave (SD) & 1 & $2016 / 11 / 14$ & $5.30(3.31)$ & $0.18(0.13)$ & $1.02(0.13)$ & $0.43(0.30)$ & $20.39(5.74)$ & $4.15(1.18)$ \\
\hline Ave (SD) & 5 & $2016 / 12 / 14$ & $6.78(2.82)$ & $0.98(1.19)$ & $1.14(0.41)$ & $0.31(0.24)$ & $23.00(9.54)$ & 4.55 (1.98) \\
\hline Ave (SD) & 6 & $2016 / 12 / 22$ & $7.89(3.83)$ & $0.21(0.15)$ & $1.15(0.30)$ & $3.26(3.64)$ & $24.22(6.32)$ & $5.14(1.28)$ \\
\hline \multicolumn{9}{|l|}{$\mathrm{B}$} \\
\hline Ave (SD) & 1 & $2017 / 1 / 9$ & $5.61(2.24)$ & $0.44(0.30)$ & $0.89(0.15)$ & $0.84(0.68)$ & $13.62(4.63)$ & $7.26(4.53)$ \\
\hline Ave (SD) & 5 & $2017 / 2 / 9$ & $7.52(5.75)$ & $0.62(0.50)$ & $1.06(0.24)$ & $0.68(0.45)$ & $15.55(6.00)$ & $9.89(8.63)$ \\
\hline Ave (SD) & 6 & $2017 / 2 / 16$ & $4.55(1.65)$ & $0.62(0.54)$ & $0.98(0.13)$ & $1.35(0.70)$ & $14.26(4.42)$ & $10.14(8.92)$ \\
\hline Week 1 & & & 0.856 & 0.107 & 0.109 & 0.200 & 0.060 & 0.160 \\
\hline Week 5 & & & 0.762 & 0.526 & 0.677 & 0.145 & 0.109 & 0.161 \\
\hline Week 6 & & & 0.069 & 0.095 & 0.234 & 0.373 & 0.013 & 0.193 \\
\hline All Ws & & & 0.307 & 0.054 & 0.135 & 0.145 & 0.0004 & 0.136 \\
\hline
\end{tabular}


weeks. The table shows that the only significant results are for MPO average concentration that was significantly higher for Cohort A compared to Cohort $\mathrm{B}$ at week $6(\mathrm{p}=0.013)$, and also when comparing all weeks combined ( $\mathrm{p}=$ 0.0004).

Table 8 compares biomarker concentrations between genders for each week and all weeks combined. It can be seen that IFN- $\gamma$ average concentration was significantly higher for males compared to females at week 6 ( $p=0.013$; see Table 6 for average concentration values). Also, TNF- $\alpha$ average concentration was significantly higher for males on week 1 , week 5 and all weeks combined ( $\mathrm{p}=$ $0.001,0.030$, and 0.00005 , respectively).

\subsection{Heart Rate Variability}

Table 9 presents Cohort A, Cohort B and both cohorts combined $(A+B)$ weekly mean and SDs (in parentheses) for HR (beats per minute), RR (breaths per minute), SDNN (milliseconds; abbreviated as $\mathrm{ms}$ ), LF $\left(\mathrm{ms}^{2}\right), \mathrm{HF}\left(\mathrm{ms}^{2}\right)$, and the ratio LF/HF. Table 10 presents statistical results ( $\mathrm{p}$ values) comparing means of each cohort and each variable between weeks while Table 11 presents statistical results between cohorts for each week and all weeks combined.

\subsubsection{Heart Rate}

From Table 9, it can be observed that Cohort B had a slightly higher average HR at the first 10 minutes HR recording of week 1 (84.2) than Cohort A (78.1). Also, Cohort $B$ had a higher weekly average $\mathrm{HR}$ over the four 10-minute recordings of week 1 (Weekly Average: 86.4 vs. 85.0). However, the weekly HR average at week 5 and week 6, as well as for all weeks combined, was higher for Cohort A. These results are not statistically significant (see Table 11). According to Table 10 (and Table 9), Cohort A had a significantly higher average HR at week 6 compared to week 1 ( 87.2 vs. 85.0; $\mathrm{p}=0.027)$. Statistical analyses between weekly HR averages for Cohort B and for both cohorts combined produce no significant result.

\subsubsection{Respiratory Rate}

Table 9 shows that Cohort B had a higher average RR at the first 10 minutes RR recording of week 1 (19.7) compared to Cohort A (14.2). Also, Cohort B had a higher weekly average RR over the four 10-minute recordings of week 1 (Weekly

Table 8. Statistical results of blood biomarker concentrations between genders for each week.

\begin{tabular}{ccccccc}
\hline F vs M & $\begin{array}{c}\text { IFN- } \gamma \\
(\mathrm{pg} / \mathrm{mL})\end{array}$ & $\begin{array}{c}\text { IL-6 } \\
(\mathrm{pg} / \mathrm{mL})\end{array}$ & $\begin{array}{c}\text { TNF-alpha } \\
(\mathrm{pg} / \mathrm{mL})\end{array}$ & $\begin{array}{c}\text { hsCRP } \\
(\mathrm{mg} / \mathrm{L})\end{array}$ & $\begin{array}{c}\text { MPO } \\
(\mathrm{ng} / \mathrm{mL})\end{array}$ & $\begin{array}{c}\text { MDA } \\
(\mathrm{ng} / \mathrm{mL})\end{array}$ \\
\hline Week 1 & 0.509 & 0.513 & 0.001 & 0.827 & 0.170 & 0.254 \\
Week 5 & 0.679 & 0.354 & 0.030 & 0.898 & 0.377 & 0.661 \\
Week 6 & 0.013 & 0.267 & 0.078 & 0.555 & 0.661 & 0.571 \\
All Ws & 0.107 & 0.468 & 0.00005 & 0.817 & 0.140 & 0.143 \\
\hline
\end{tabular}




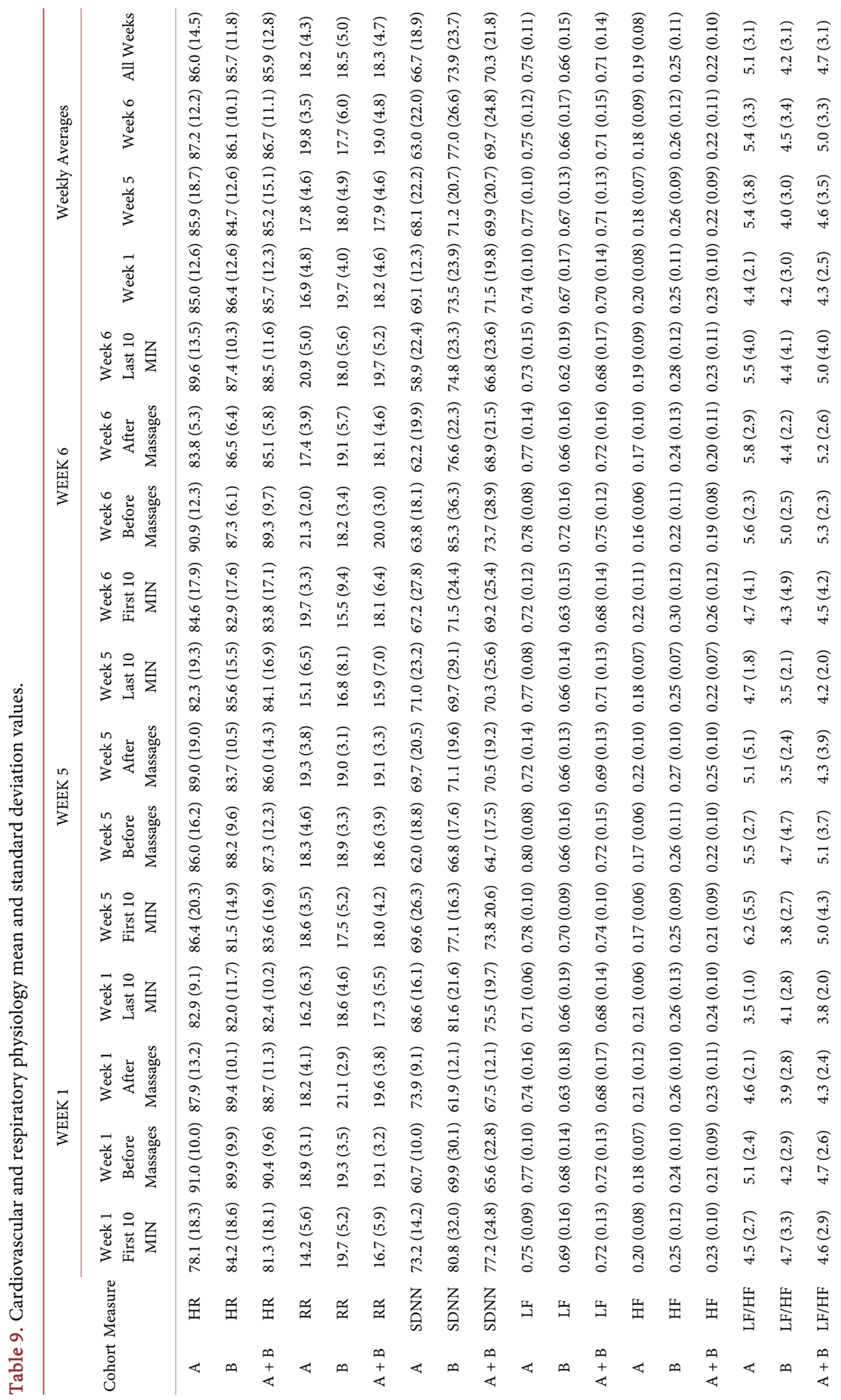


Table 10. Cardiovascular and respiratory physiology statistical results within cohorts.

\begin{tabular}{|c|c|c|c|c|}
\hline Cohort & Measure & W1 vs W5 & W1 vs W6 & W5 vs W6 \\
\hline A & HR & 0.287 & 0.027 & 0.247 \\
\hline B & HR & 0.055 & 0.061 & 0.191 \\
\hline$A+B$ & HR & 0.084 & 0.256 & 0.113 \\
\hline A & $\mathrm{RR}$ & 0.269 & 0.004 & 0.023 \\
\hline B & $\mathrm{RR}$ & 0.071 & 0.100 & 0.426 \\
\hline$A+B$ & $\mathrm{RR}$ & 0.138 & 0.148 & 0.058 \\
\hline A & SDNN & 0.332 & 0.026 & 0.017 \\
\hline B & SDNN & 0.108 & 0.212 & 0.094 \\
\hline$A+B$ & SDNN & 0.084 & 0.186 & 0.161 \\
\hline A & LF & 0.013 & 0.320 & 0.285 \\
\hline B & LF & 0.430 & 0.264 & 0.233 \\
\hline$A+B$ & LF & 0.136 & 0.229 & 0.200 \\
\hline A & $\mathrm{HF}$ & 0.013 & 0.084 & 0.297 \\
\hline B & $\mathrm{HF}$ & 0.108 & 0.221 & 0.398 \\
\hline$A+B$ & $\mathrm{HF}$ & 0.245 & 0.342 & 0.298 \\
\hline A & $\mathrm{LF} / \mathrm{HF}$ & 0.013 & 0.124 & 0.280 \\
\hline B & $\mathrm{LF} / \mathrm{HF}$ & 0.108 & 0.351 & 0.297 \\
\hline$A+B$ & $\mathrm{LF} / \mathrm{HF}$ & 0.210 & 0.210 & 0.206 \\
\hline
\end{tabular}

Table 11. Cardiovascular and respiratory physiology statistical results between cohorts (A vs. B).

\begin{tabular}{cccccc}
\hline Cohort & Measure & Week 1 & Week 5 & Week 6 & All Weeks \\
\hline A vs B & HR & 0.678 & 0.930 & 0.708 & 0.828 \\
A vs B & RR & 0.028 & 0.813 & 0.131 & 0.589 \\
A vs B & SDNN & 0.818 & 0.594 & 0.034 & 0.124 \\
A vs B & LF & 0.090 & 0.002 & 0.013 & 0.0001 \\
A vs B & HF & 0.088 & 0.001 & 0.014 & 0.00001 \\
A vs B & LF/HF & 0.077 & 0.002 & 0.019 & 0.00002 \\
\hline
\end{tabular}

Average: 19.7 vs. 16.9$)$ and this result was significant ( $p=0.028$; Table 11$)$. Similarly, the weekly RR average at week 5 was higher for Cohort B but the reverse was true for week 6 (not significant in both cases). Comparing the total average RR between cohorts, it can be seen that Cohort B has a higher total average RR than Cohort A but not by much (18.5 vs. 18.2, not significant). According to Table 10 (and Table 9), Cohort A had a significantly higher weekly average RR at week 6 compared to week 1 and week 5 (19.8 vs. 16.9; $\mathrm{p}=0.004 ; 19.8$ vs. 17.8; $\mathrm{p}=0.023$ ). There was no significant result between weeks for Cohort B and for both cohorts combined. 


\subsubsection{SDNN}

According to Table 10 (and Table 9), Cohort A had a significantly lower weekly average SDNN at week 6 compare to week 1 and week 5 (63.0 vs. 69.1, $\mathrm{p}=0.026$; 63.0 vs. $68.1 ; \mathrm{p}=0.017$, respectively). There are no significant values for Cohort $\mathrm{B}$ and for both cohorts combined. According to Table 11, Cohort B had a significantly higher average SDNN values than Cohort A at week 6 (77.0 vs. 63.0; $\mathrm{p}=0.034)$.

\subsubsection{LF}

According to Table 10 and Table 9, Cohort A had a significantly higher weekly $\mathrm{LF}$ average value at week 5 than at week $1(0.77$ vs. $0.74 ; \mathrm{p}=0.013)$. Comparing Cohort A and Cohort B, Table 11 shows that at week 5, week 6 and for all weeks combined Cohort $\mathrm{A}$ had a significantly higher average LF value than Cohort $\mathrm{B}$ ( 0.77 vs. $0.67, \mathrm{p}=0.002 ; 0.75$ vs. 0.66 . $\mathrm{p}=0.013 ; 0.75$ vs. $0.66, \mathrm{p}=0.0001$, respectively).

\subsubsection{HF}

Table 9 and Table 10 show that Cohort $A$ has a lower average HF value at week 5 compared to week $1(0.18$ vs. $0.20, \mathrm{p}=0.013)$. Cohort $\mathrm{B}$ has higher average HF value at week 5, week 6 and for all weeks combined compared to Cohort A (0.26 vs. $0.18, \mathrm{p}=0.001 ; 0.26$ vs. $0.18, \mathrm{p}=0.014 ; 0.25$ vs. $0.19, \mathrm{p}=0.00001$, respectively).

\subsubsection{LF/HF}

For LF/HF the results are similar as with LF, with week 5, week 6 and all weeks combined showing significantly higher average LF/HF value for Cohort A according to Table 9 and Table 10 ( $\mathrm{p}=0.002,0.019$, and 0.00002, respectively). Also, Table 11 shows that Cohort A has significantly higher average LF/HF value at week 5 compared to week 1 ( 5.37 vs. $4.43 ; \mathrm{p}=0.013$ ). This is also similar to LF.

\section{Discussion}

This exploratory study was conducted to extend a prior pilot study which examined the wellbeing effects of grounding on massage therapists being grounded while they performed their massage work [5]. This current study extended those findings by using a larger set of assessments and providing grounding during the therapist's night's sleep in addition to them being grounded while they performed their massage work.

While there are numerous findings to discuss, we begin with the random assignment process itself as it has a bearing on the subsequent discussion. Even though participants were randomly assigned to each group, the randomization process did not yield equitable cohorts in terms of gender, although this difference was not statistically significant (Table 1 ). There were several significant cohort baseline differences for some of the physiological data. First, both systolic blood viscosity (SBV) and diastolic blood viscosity (DBV) were statistically 
higher for Cohort A at week 1 compared to Cohort B at week 1 (Table 2). This could indicate a higher level of systemic inflammation on average in the participants of Cohort A at the start of their participation compared to Cohort B. Table 3 shows that males had significantly higher SBV and DBV than females for all weeks (except for SBV at week 5). Since Cohort B had only one male participant, this result implies that the lower blood viscosity for Cohort B at all weeks seen in Table 2 can be attributed at least partially to the fact that this cohort had fewer males. Also, Table 3 shows significant decreases in female blood viscosity at week 6 compared to week 5, for both SBV and SDV, while there were no significant results for male participants. This result implies that the significant decrease in SBV for Cohort B at week 6 compared to week 5 seen in Table 2 is most likely due to the female participants. From these results, it is clear that gender composition had an effect on cohorts' average blood viscosity. There is a known dependence of the oxygen delivery index (ODI) to hematocrit and SBV and it is also well known that females have a slightly lower hematocrit in general [13]. Since Cohort B was almost exclusively composed of females while Cohort A had the same number of females and males, it is likely that the difference in gender composition between cohorts contributed to blood viscosity (both SBV and DBV) to be slightly lower for Cohort B. However, it is not clear that gender composition was to only explanation for the lower blood viscosity of Cohort B.

A second indication that the cohorts were different from the start comes from blood biomarkers (Tables 4-8). From Table 5, TNF- $\alpha$ and MPO average concentrations for all weeks combined were statistically higher for Cohort A compared to Cohort B (1.36 vs. 0.99, $\mathrm{p}=0.009,21.1$ vs. $15.4 ; \mathrm{p}=0.003$, respectively; all weeks combined average concentrations were calculated from Table 4). Additionally, TNF- $\alpha$ average concentration at week 1 was significantly higher for Cohort A compared to Cohort B. These results point toward basic cohort differences in biomarkers at the start of their participation as well as during the entire time of their participation with Cohort A having a tendency toward higher levels of biomarker average concentrations. On the other hand, Table 7 shows that females from Cohort A had a significantly higher MPO average concentration for all weeks compared to females from Cohort $B(p=0.0004)$. This last result indicates that there is probably another factor than gender making Cohort $\mathrm{A}$ higher in blood viscosity and biomarkers than Cohort B. We propose that this other factor could be time of the year as will be explained below. Also, from Table 8 (and Table 6 for average concentrations), IFN- $\gamma$ average concentration was significantly higher for males at week 6 and male TNF- $\alpha$ average concentration was also significantly higher than that of females for week 1, week 5 and for all weeks combined. These results are other indications that male physiology is different than that of females and that gender composition contributed to differences in blood viscosity and biomarkers between cohorts.

A third line of evidence for basic physiological differences between cohorts comes from HRV analysis. LF, HF and LF/HF average values between cohorts 
for all 3 weeks combined are very significantly different $(\mathrm{p}=0.0001,0.00001$ and 0.0002, respectively; see Tables 9-11). Also, for all three variables, Cohort A values are significantly different at week 5 and week 6 . These results mean that each cohort autonomic nervous system (ANS) reacted differently during the duration of their participation. Why would Cohort A start and stay with higher levels of inflammation and stress compared to Cohort B during the entire time of their participation? We see two potential explanations. One explanation is because of difference in gender composition as already explained, however, we have seen evidence that this explanation cannot be the whole story (see Table 7 and related explanations). Another plausible explanation is time of the year. Cohort A participation started 11/14/2016 and ended on 12/22/2016. So, these participants started 10 days before Thanksgiving (11/24/16) and their participation ended just a few days before Christmas. They were just in the middle of the holiday season. No doubt that these participants experienced extra stress due to Thanksgiving and then Christmas preparation on top of their regular work schedule. We also have to add to that the stress these participants accumulated after working for months at end. They were surely looking forward to their holiday break. The research coordinators said they had comments about both of these situations. On the other end, Cohort B participation started on 1/4/2017 and ended on 2/15/2017, just after the Holidays. They had time to enjoy the Holidays without working and it is very likely that the participants of this cohort started refreshed from their time off and were ready to go to work. It is quite normal to expect that their level of stress would be lower than that of Cohort A, and that is what we observe in the data i.e. for all weeks combined, LF and LF/HF are significantly lower for Cohort B, while HF is significantly higher for Cohort B compared to Cohort A. In light of these very significant differences between cohorts, extra attention was given to each cohort and their difference in gender composition (for blood viscosity and biomarkers).

Coming back to blood viscosity, the normal range for SBV is between 3.7 and $4.4 \mathrm{cP}$ while it is between 8.9 and $12.4 \mathrm{cP}$ for DBV. According to results presented in Table 2, Cohort A started their first week of participation with normal levels of blood viscosity going down to lower levels during their participation time while Cohort B started with lower than normal levels of blood viscosity at week 1, stayed lower than normal for the entire duration of their participation, and became even lower at week 6 (the end of their participation time, after being ungrounded for one week). Table 2 also shows that SBV was significantly lower at week 6 compared to week 1 for Cohort A while a significant decrease in SBV was observed at week 6 compared to week 5 for Cohort B. These results show a tendency for blood viscosity to be lower at week 6 and suggest that the effect of grounding continue to improve blood viscosity at least one week after the end of the 4 -week grounding period. Finally, Table 3 shows significantly lower SBV and DBV at week 6 for female participants but not for males, reinforcing the hypothesis that female participants were the reason for the decrease in blood 
viscosity at week 6 seen in Table 2 for Cohort B. However, gender composition cannot explain why SBV was significantly lower at week 6 compared to week 1 for Cohort A in Table 2, suggesting that gender composition is not involved in the production of this result.

Turning our attention to blood biomarkers, Table 4 shows that for Cohort A IFN- $\gamma$ average concentration was significantly higher at week 6 compared to week 1 and week 5. The same table shows that for Cohort B TNF- $\alpha$ average concentration was significantly higher at week 6 compared to week 1 and hsCRP average concentration was higher at week 6 compared to week 1 and week 5 . For both cohorts combined, TNF- $\alpha$ average concentration was higher at week 6 than at week 1 and also hsCRP concentration was higher at week 6 compared to week 5 and week 1 . Since IFN- $\gamma$, TNF- $\alpha$ and hsCRP are markers of inflammation, these results suggest a tendency for inflammation to increase markedly one week after participants stopped grounding (end of week 6, when blood samples were taken for the last time). These results seem to contradict blood viscosity results. However, IFN- $\gamma$, TNF- $\alpha$ and hsCRP are direct markers of inflammation i.e. they are part of the physiological mechanisms that promote inflammation inside the body while blood viscosity is a measure of systemic effects of grounding on the blood. It is possible that blood viscosity continues to improve because of the presence of extra electrons attached to red blood cells (RBCs) even after not being grounded for some time, while deeper inside the body the mechanisms of inflammation are already in gear. If that hypothesis is true, when the electron reserve is depleted and the absolute value of the zeta potential of RBCs decreases, blood viscosity would then increase [22]. We just don't know how much time it takes for electron depletion to happen. An increase in low density lipoprotein-cholesterol (LDL-C) can decrease erythrocyte deformability by increasing the cholesterol-to-phospholipid ratio at the erythrocyte membrane, resulting in an increase in SBV while a decrease in LDL-C would produce the opposite result. Other factors decreasing erythrocyte membrane deformability are glucose, osmolality and dehydration. Hematocrit also has an important effect on SBV. SBV increases exponentially at high hematocrit [16]. Another possible explanation is that grounding may decrease hematocrit in grounded participants and that effect may override or precede the increase in inflammation by some weeks resulting in temporary small decreases in SBV as seen in Table 2 and Table 3. A third possible explanation is that grounding may increase deformability of erythrocytes (because of the increase in absolute zeta potential due to extra electrons in the body) for some time even after ungrounding [22]. Since the number of participants in each cohort was small, research with more participants (and more methods of analysis) is needed in order to determine which of the 3 hypotheses (or combination of hypotheses) is (are) correct.

Table 9 and Table 10 show results for HR. According to these tables, Cohort A had a significantly higher average HR at week 6 compared to week 1, an indication of increased level of stress above the level of stress at the beginning of the 
study. This result is to be compared with Table 4 biomarker results of an increase in IFN- $\gamma$ concentration for Cohort A at week 6 compared to week 1 and week 5. We can infer that the increased stress seen in HR at week 6 for Cohort A is correlated to an increase in inflammation. On the other hand, Cohort B week $5 \mathrm{HR}$ was lower than that at week 1 or week 6, possibly suggesting a relaxation effect after 4 weeks of grounding (although not significant). Cohort A HR weekly average kept increasing suggesting an increase in stress over time culminating in the highest stress level at the end of week 6. These opposite responses in HR between cohorts to grounding (at the end of week 5, after 4 weeks of grounding) give more credibility to the hypothesis that Cohort A was under increased stress during the time of participation while this was not the case for Cohort B which responded by greater relaxation as observed in previous studies [23] [24] [25].

From these same tables, it can be seen that Cohort B had a significantly higher weekly average RR over the four 10-minute recordings of week 1 compared to Cohort A ( $p=0.028$; Table 11). Remember that week 1 was an ungrounded week and so this result again points toward a difference between these two cohorts at the start of their participation. Cohort A had a significantly higher weekly average RR at week 6 compared to week 1 and week 5 . A slower RR is usually an indication of a more relaxed state, parasympathetic system (PNS) activation, and deeper respiration. On the other hand, faster RR is related to increased activation of the sympathetic nervous system (SNS) and stress. The present results suggest increasing levels of stress as time pass during Cohort A participation, culminating with the higher stress level at week 6 while the opposite is true for Cohort B. This result reinforces the results obtained for HR suggesting a high level of stress at the end of Cohort A participation time (week 6) compared to week 1. For Cohort B, RR decreased after 4 weeks of grounding (at week 5, although not significantly), in agreement with the results obtained for HR. This agreement between HR and RR is not surprising since there is a known physiological mechanism linking the two through brainstem networks and respiratory sinus arrhythmia (RSA) [26] [27].

According to the Task Force [20], SDNN, the square root of variance between inter-beat intervals, reflects all the cyclic components responsible for variability in the period of recording (10 minutes in our case). It has been established that low SDNN (or HRV) is a factor increasing the risk of cardiovascular problems including heart attacks [28] [29]. Consequently, an increase in HRV is considered a positive outcome [30]. According to Table 9 and Table 10, Cohort A had a significantly lower weekly average SDNN at week 6 compare to week 1 and week 5. On the other hand, at week 6 Cohort B had a significantly higher weekly SDNN average than Cohort A ( $\mathrm{p}=0.034$, Table 11). These results are consistent with previous results for $\mathrm{HR}$ and $\mathrm{RR}$ showing an increase in stress one week after the end of Cohort A's participation time and the opposite for Cohort B.

According to Tables 9-11, Cohort A had a significantly higher weekly LF average value at week 5 compared to week 1 . Comparing Cohort A and Cohort B, 
the tables show that at week 5 and week 6, Cohort A had a significantly higher average LF value than Cohort $B$ ( $p=0.002$ and 0.013 , respectively); this is also true for all weeks combined $(\mathrm{p}=0.0001)$. In general, the consensus is that LF contains information from both the sympathetic Nervous system (SNS) and the parasympathetic nervous system (PNS). Some researchers came to the conclusion that LF can be used as a marker of SNS function during the recording of the change from supine (when LF would be considered to contain information predominantly from the PNS) to sitting position (when LF would be considered to contain information predominantly from the SNS) in healthy people [31]. A recent heart rate control model provides support to this notion as a mathematical model developed to simulate LF requires in the HR control loop sympathetic cardiac-related oscillations generated in the brain stem [32]. Since our participants were standing (before, during or after massages) we take LF to predominantly contain information on the SNS and stress. We then view the increase in LF at week 5 compare to week 1 for Cohort A to be an indicator of increased stress. These results for $L F$ are in agreement with results from $H R, R R$, and SDNN.

Regarding HF, there is wide agreement that this variable is closely related to vagal tone and PNS function [20] [33]. According to Tables 9-11, Cohort A had a significantly lower average HF value at week 5 compared to week 1 ( $p=0.013$ ), an indication of increased stress. On the other hand, Cohort B had a significantly higher average HF than Cohort A at week 5, week 6 and for all weeks combined ( $\mathrm{p}=0.001,0.014$ and 0.00001 , respectively). We note also that at week 5 Cohort $B$ had an increase in HF compare to week 1 (although not significant). These results are in accordance with results obtained in a previous study where HF increased significantly during a 40-minute grounding period compared to an ungrounded group [23]. In this previous study, the increase in HF during the grounding period was more than double that of the non-grounded group. It can be noted that as soon as the grounding period ended, HF started to decrease. This phenomenon could explain why we do not see a statistically significant increase in average HF for Cohort B after 4 weeks of grounding (week 5). In effect, the weekly four10-minutes periods (first 10 minutes after putting the recorder on, 10-minutes before the first massage, first 10-minutes after massages, and last 10-minutes before taking off the recorder) used to calculate HRV parameters are times when the participants were not grounded. Consequently, the decrease in HF starting immediately after ungrounding seen in a previous study could explain the present result for Cohort B. Measuring participants during grounding may have resulted in more significant results. However, the present study was designed in part to address long term positive effects of grounding (for 4 weeks) and in part to determine if the positive effects last after ungrounding for one week. Nevertheless, the fact that Cohort B HF increases at week 5 compared to week 1 while the opposite is true for Cohort A, resulting in significant differences between cohorts for week 5, week 6 and all weeks combined, is a confirmation 
that Cohort A was under abnormally high levels of stress and that Cohort B was more relaxed. Again, these results are similar and support the results obtain with the previous variables (HR, RR, SDNN and LF).

According to the Task Force [20], LF/HF is the best variable to determine the ratio of vagal tone to SNS activation. Consequently, it is not surprising to see that LF/HF results are very similar to those for HF in this study since our participants were recorded while standing (i.e. LF contained mainly information on SNS). It can be seen from Tables 9-11, that the same statistical results are significant for both variables. In effect if HF correlates with PNS and LF with SNS, the ratio on the two (LF/HF) should result in very similar statistical results with high and low values inverted compared to HF (i.e. the low values of HF become high values of $\mathrm{LF} / \mathrm{HF}$ and vice-versa). This is exactly what we observe. According to Table 9 and Table 11, at week 5, week 6, and for all weeks combined Cohort $B$ had a significantly lower mean average LF/HF than Cohort A ( $p=0.002,0.019$ and 0.00002, respectively). Also, Cohort A had a significantly higher average LF/HF value at week 5 compared to week 1 , an indication of increased stress. Again, these results are consistent with all other HRV related results.

A limitation of this study is the modest number of participants. For this reason, we highlighted in blue probabilities between 0.05 and 0.1 as possibly of interest to help design future studies with a larger number of participants. Also, this study suggests the importance of gender differences and the time of the year for doing such an experiment. It is best to make sure gender composition is similar in all cohorts and it is best to avoid setting up an experiment close to holidays. Another limitation is that the present design did not allow us to investigate independently the effects of BMI from those due to gender. In addition, physiological data (HR, HRV) were obtained in participants allowed to move freely during measurement intervals, and variations in motor activity and posture may have affected these measures. The level of physical activity (intensity of motion and exertion) during the massage event may also differ between participants, thus influencing HR and respiratory rate after the massage. Future studies could examine HRV during periods of rest (e.g., sitting quietly in a chair) to address these issues. In futures studies, an assessment of general stress level for several cohorts starting at different time points may confirm the present conclusions regarding the differences at baseline. Finally, it would be interesting to assess if perceived level of stress correlates with changes in biomarkers.

\section{Conclusion}

This exploratory study showed that grounding massage therapists while they performed massages and at night reduces stress as indicated by $H R, R R, L F, H F$ and LF/HF. It also showed that the lowering effect of grounding on blood viscosity lasts for at least one week after ungrounding, with systolic blood viscosity becoming significantly lower at the end of the study as compared to the initial pre-intervention value. Inflammation markers (IFN- $\gamma, \mathrm{TNF}-\alpha$, and hsCRP) 
increased rapidly after ungrounding, within a week, suggesting the importance of grounding on a regular basis, preferably daily. Abnormally stressful situations lasting for long periods of time can partially decrease the benefits of grounding, but not eliminate them. This study's findings suggest that grounding is beneficial for massage therapists in several domains relevant to health and wellbeing

\section{Acknowledgements}

The authors wish to thank Jennifer Johnson, Director of Services at the Chopra Center for Wellbeing, and the staff at the Chopra Center for supporting this project. We are particularly grateful to the massage therapists at the Chopra Center for their extensive time and commitment to this study. We acknowledge Daniel J Cho, Director of Rheovector LLC, for blood viscosity determinations. This project was funded by Earth FX, Inc., and the grounding products were donated by earthing.com.

\section{Disclosures}

One or more authors have received funding and/or advisory fees from health companies for other projects. P.J.M. is director of research at the Chopra Foundation. S.P. is employed by the Chopra Center and L.W. is an employee of the Chopra Foundation. D.C. is a co-founder and a co-owner of the Chopra Center. G.C. is a consultant for Earth FX.

\section{References}

[1] Chevalier, G., Sinatra, S.T., Oschman, J.L., Sokal, K. and Sokal, P. (2012) Earthing: Health Implications of Reconnecting the Human Body to the Earth's Surface Electrons. Journal of Environmental and Public Health, 2012, Article ID: 291541. https://doi.org/10.1155/2012/291541

[2] Oschman, J.L., Chevalier, G. and Brown, R. (2015) The Effects of Grounding (Earthing) on Inflammation, the Immune Response, Wound Healing, and Prevention and Treatment of Chronic Inflammatory and Autoimmune Diseases. Journal of Inflammation Research, 8, 83-96. https://doi.org/10.2147/JIR.S69656

[3] Oschman, J.L., Chevalier, G. and Ober, A.C. (2015) Biophysics of Earthing (Grounding) the Human Body. In: Rosch, P.J., Ed., Bioelectromagnetic and Subtle Energy Medicine, 2nd Edition, CRC Press, New York, 427-448.

[4] Lyons, N. (2016) 3 Things You Should Know Before Becoming a Massage Therapist. http://www.massagetherapyschoolsinformation.com/becoming-a-massage-therapist/

[5] Zucker, M. (2016) Goodbye Pain and Inflammation: Massage Table Grounding Pad Relieves Therapist's Overuse Injuries. Dermascope, May Issue. http://www.dermascope.com/scope-this/goodbye-pain-and-inflammation-massageta-

ble-grounding-pad-relieves-therapist-s-overuse-injuries?print=1\&tmpl=component \#.WdZxQjBryUk

[6] Omoigui, S. (2015) The Biochemical Origin of Pain-Proposing a New Law of Pain: The Origin of All Pain is Inflammation and the Inflammatory Response. Part 1 of 3-A Unifying Law of Pain. Medical Hypotheses, 69, 70-82. https://doi.org/10.1016/j.mehy.2006.11.028 
[7] Omoigui, S. (2015) The Biochemical Origin of Pain: The Origin of All Pain Is Inflammation and the Inflammatory Response. Part 2 of 3-Inflammatory Profile of Pain Syndromes. Medical Hypotheses, 69, 1169-1178. https://doi.org/10.1016/j.mehy.2007.06.033

[8] Louati, K. and Berenbaum, F. (2015) Fatigue in Chronic Inflammation-A Link to Pain Pathways. Arthritis Research \& Therapy, 17, 254.

https://doi.org/10.1186/s13075-015-0784-1

[9] Jones, R. (2009) Investing in Yourself. Massagetherapy.com. http://www.massagetherapy.com/articles/index.php/article_id/1774/Investing-In-Y ourself

[10] Oschman, J.L. (2016) Earthing: A New Perspective on the Cause and Prevention of therapist Burnout. Massage \& Body Work, March/April, 75-81.

[11] Brown, C.A. and Lilford, R.J. (2006) The Stepped Wedge Trial Design: A Systematic Review. BMC Medical Research Methodology, 8, 6-54. https://doi.org/10.1186/1471-2288-6-54

[12] Hussey, M.A. and Hughes, J.P. (2007) Design and Analysis of Stepped Wedge Cluster Randomized Trials. Contemporary Clinical Trials, 28, 182-191. https://doi.org/10.1016/j.cct.2006.05.007

[13] Pop, G.A.M., Duncker, D.J., Gardien, M., Vranckx, P., Versluis, S., Hasan, D. and Slager, C.J. (2002) The Clinical Significance of Whole Blood Viscosity in (Cardio)vascular Medicine. Netherlands Heart Journal, 10, 512-516.

[14] Tamariz, L.J., Young, J.H., Pankow, J.S., Yeh, H.C., Schmidt, M.I, Astor, B. and Brancati, F.L. (2008) Blood Viscosity and Hematocrit as Risk Factors for Type 2 Diabetes Mellitus: The Atherosclerosis Risk in Communities (ARIC) Study. American Journal of Epidemiology, 168, 1153-1160. https://doi.org/10.1093/aje/kwn243

[15] Rafnsson, S., Deary, I.J., Whiteman, M.C., Rumley, A., Lowe, G.D.O. and Fowkes, F.G.R. (2010) Haemorheological Predictors of Cognitive Decline: The Edinburgh Artery Study. Age and Ageing, 39, 217-222. https://doi.org/10.1093/ageing/afp227

[16] Cho, Y-I. and Cho, D.J. Hemorheology and Microvascular Disorders. Korean Circulation Journal, 41, 287-295. https://doi.org/10.4070/kcj.2011.41.6.287

[17] Larsen, P. and Holsworth, R. (2012) Measuring Blood Viscosity to Improve Patient Outcomes. Townsend Letter: The Examiner of Alternative Medicine. http://www.townsendletter.com/Jan2012/measureblood0112.html

[18] Marton, Z., Kesmarky, G., Vekasi, J., Cser, A., Russai, R., Horvath, B. and Toth, K. (2001) Red Blood Cell Aggregation Measurements in Whole Blood and in Fibrinogen Solutions by Different Methods. Clinical Hemorheology and Microcirculation, 24, 75-83.

[19] Chen, W.-L., Tsai, T.-H., Huang, C.-C., Chen, J.-H. and Kuo, C.-D. (2009) Heart Rate Variability Predicts Short-Term Outcome of Successfully Resuscitated Patients with Out-of-Hospital Cardiac Arrest. Resuscitation, 80, 1114-1118. https://doi.org/10.1016/j.resuscitation.2009.06.020

[20] Task Force of the European Society of Cardiology and the North American Society of Pacing and Electrophysiology (1996) Heart Rate Variability: Standards of Measurement, Physiological Interpretation, and Clinical Use. European Heart Journal, 17, 354-381. https://doi.org/10.1093/oxfordjournals.eurheartj.a014868

[21] Vivonoetics (2017) VivoSense ${ }^{\circledast}$ User Manual-Heart Rate Variability Professional Edition. Newport Beach, CA. 
[22] Chevalier, G., Sinatra S.T., Oschman, J.L. and Delany, R.M. (2013) Earthing (Grounding) the Human Body Reduces Blood Viscosity-A Major Factor in Cardiovascular Disease. Journal of Alternative and Complementary Medicine, 19, 102-110. https://doi.org/10.1089/acm.2011.0820

[23] Chevalier, G. and Sinatra, S.T. (2011) Emotional Stress, Heart Rate Variability, Grounding, and Improved Autonomic Tone: Clinical Applications. Integrative Medicine, 10, 16-21.

[24] Chevalier, G., Mori, K. and Oschman, J.L. (2006) The Effects of Earthing (Grounding) on Human Physiology. European Biology and Bioelectromagnetics, 600-621.

[25] Chevalier, G. (2010) Changes in Pulse Rate, Respiratory Rate, Blood Oxygenation, Perfusion Index, Skin Conductance, and Their Variability Induced During and After Grounding Human Subjects for 40 Minutes. Journal of Alternative and Complementary Medicine, 16, 81-87. https://doi.org/10.1089/acm.2009.0278

[26] Murata, A. (1992) Effects of Respiration on Heart-Rate Variability Measures. Electronics and Communications in Japan, 75, 107-116. https://doi.org/10.1002/ecjc.4430750210

[27] Garcia, A.J., Koschnitzky, J.E., Dashevskiy, T. and Ramirez, J.-M. (2013) Cardiorespiratory Coupling in Health and Disease. Autonomic Neuroscience: Basic and Clinical, 175, 26-37. https://doi.org/10.1016/j.autneu.2013.02.006

[28] Wolf, M.M., Varigos, G.A., Hunt, D. and Sloman, J.G. (1978) Sinus Arrhythmia in Acute Myocardial Infarction. The Medical Journal of Australia, 2, 52-53.

[29] Sinnecker, D., Dommasch, M., Steger, A., Barkefekd, A., Hoppmann, P., Muller, A., Gebhardt, J., Barthel, P., Hnatkova, K., Huster, K.H., Laugwitz, K.-L., Malik, M. and Schmidt, G. (2016) Expiration-Triggered Sinus Arrhythmia Predict Outcome in Survivors of Acute Myocardial Infarction. Journal of the American College of Cardiology, 67, 2213-2220. https://doi.org/10.1016/j.jacc.2016.03.484

[30] Passi, R., Doheny, K.K., Gordin, Y., Hinssen, H. and Palmer, C. (2017) Electrical Grounding Improves Vagal Tone in Preterm Infants. Neonatology, 112, 187-192. https://doi.org/10.1159/000475744

[31] Perini, R. and Veicsteinas, A. (2003) Heart Rate Variabiity and Autonomic Activity at Rest and During Exercise in Various Physiological Conditions. European Journal of Applied Physiology, 90, 317-325. https://doi.org/10.1007/s00421-003-0953-9

[32] Dvir, H., Bobrovsky, B.Z. and Gabbay, U. (2013) A Novel Heart Rate Control Model Provides Insights Linking LF-HRV Behavior to the Open-Loop Gain. International Journal of Cardiology, 168, 287-293. https://doi.org/10.1016/j.ijcard.2012.09.073

[33] Pichon, A., de Bisschop, C., Diaz, V. and Denjean, A. (2005) Parasympathetic Airway Response and the Heart Rate Variability Before and at the End of Methacholine Challenge. Chest, 127, 23-29. https://doi.org/10.1378/chest.127.1.23 